\title{
A NEW INVARIANT OF HOMOTOPY TYPE AND SOME DIVERSE APPLICATIONS
}

\author{
BY DANIEL H. GOTTLIEB ${ }^{1}$
}

Communicated by J. Milnor, December 14, 1964

Let $X$ be a connected, locally finite simplicial polyhedron. Let $X^{X}$ be the space of maps from $X$ to $X$ with the compact-open topology. Let $x_{0} \in X$ be taken as a base point in $X$, then the evaluation map $p: X^{x} \rightarrow X$ defined by $p(f)=f\left(x_{0}\right)$ for $f \in X^{X}$ is continuous. Now $p$ induces the homomorphism

$$
p_{*}: \pi_{1}\left(X^{X}, 1_{X}\right) \rightarrow \pi_{1}\left(X, x_{0}\right),
$$

where $1_{X} \in X^{x}$ is the identity map. Hence $p_{*} \pi_{1}\left(X^{x}, 1_{X}\right)$ is a subgroup of the fundamental group of $\left(X, x_{0}\right)$.

Proposition 1. $p_{*} \pi_{1}\left(X^{X}, 1_{X}\right)$ considered as a subgroup of $\pi_{1}\left(X, x_{0}\right)$ is an invariant of homotopy type.

In [2], this invariant is studied and theorems are obtained which bear on the study of $X^{x}$, groups of homeomorphisms, homological group theory and knot theory. Most of these results come from the following theorem.

TheOREM 2. Let $X$ have the homotopy type of a compact, connected polyhedron with nonzero Euler-Poincaré number. Then $p_{*} \pi_{1}\left(X^{x}, 1_{X}\right)$ $=0$.

The proof of this employs Nielsen-Wecken fixed-point class theory ([1] and [5]).

Let $G(X)$ be the group of homeomorphisms of a manifold $X$, and let $G_{0}(X)$ be the isotropy group over $x_{0}$. Then there is an exact sequence [3]

$$
\cdots \rightarrow \pi_{i}\left(G_{0}(X), 1_{X}\right) \stackrel{i_{*}}{\rightarrow} \pi_{i}\left(G(X), 1_{X}\right) \stackrel{p_{*}^{\prime}}{\rightarrow} \pi_{i}\left(X, x_{0}\right) \rightarrow \cdots,
$$

where $p^{\prime}: G(X) \rightarrow X$ is the evaluation map.

Corollary 3. Let $X$ be as in Theorem 2. Then $p_{*}^{\prime} \pi_{1}\left(G(X), 1_{X}\right)=0$. In particular, if $\pi_{2}\left(X, x_{0}\right)=0$, then $i_{*}: \pi_{1}\left(G_{0}(X), 1_{X}\right) \cong \pi_{1}\left(G(X), 1_{X}\right)$.

This follows because $p_{*}^{\prime} \pi_{1}\left(G(X), 1_{X}\right) \subseteq p_{*} \pi_{1}\left(X^{X}, 1_{X}\right)$.

\footnotetext{
1 This work was partially supported by NSF Grant 1908.
} 
THEOREM 4. If $X$ is an aspherical polyhedron, then $p_{*} \pi_{1}\left(X^{X}, 1_{X}\right)$ $=Z\left(\pi_{1}\left(X, x_{0}\right)\right)$, the center of $\pi_{1}\left(X, x_{0}\right)$.

Theorems 2 and 4 combine to give us the following corollaries:

CoRollary 5. If $X$ has the same homotopy type as a compact, connected, aspherical polyhedron with nonzero Euler-Poincaré number, then $Z\left(\pi_{1}\left(X, x_{0}\right)\right)=0$.

John Stallings, in [4], has put this result in a purely algebraic setting; namely, if a group $G$ admits a finite resolution, then, if $Z(G)$ is nontrivial, the (suitably defined) Euler-Poincaré number is zero.

Alexander's Duality and the last corollary gives us a result suggested by L. P. Neuwirth.

Corollary 6. Suppose that $X$ is a subcomplex of the $n$-sphere $S^{n}$ whose Euler characteristic is different from that of $S^{n}$. If $S^{n}-X$ is connected and aspherical, then $\pi_{1}\left(S^{n}-X\right)$ has no center.

Finally, we are able to show the following:

Theorem 7. If $X$ is aspherical, then

$$
\begin{aligned}
& \pi_{1}\left(X^{X}, 1_{X}\right) \cong Z\left(\pi_{1}\left(X, x_{0}\right)\right) \\
& \pi_{n}\left(X^{X}, 1_{X}\right) \cong 0, \quad n>1 .
\end{aligned}
$$

Note that Theorem 7 and Theorem 2 give us:

CoROllary 8. If $X$ has the homotopy type of an aspherical compact polyhedron whose Euler characteristic is different from zero, then the identity component of $X^{X}$ is contractible.

\section{BiBLIOGRAPHY} 339.

1. Jaing Bo-ju, Estimation of the Nielsen numbers, Chinese Math. 5 (1964), 330-

2. D. H. Gottlieb, A certain subgroup of the fundamental group, Amer. J. Math. (to appear).

3. G. S. McCarty, Homeotopy groups, Trans. Amer. Math. Soc. 106 (1963), 293-304.

4. John Stallings, Centerless groups-An algebraic formulation of Gottlieb's theorem (to appear).

5. F. Wecken, Fixpunktklassen. I, Math. Ann. 117 (1941), 659-671; II, ibid. 118 (1941), 216-234; III, ibid. 118 (1942), 544-577.

Institute for Defense Analyses AND UNIVERSITY OF ILLINOIS 